\title{
Modeling of Criteria Air Pollutant Emissions from Selected Nigeria Petroleum Refineries
}

\author{
Temitayo Oladimeji ${ }^{1}$, Jacob Sonibare ${ }^{2}$, Moradeyo Odunfa ${ }^{3}$, Augustine Ayeni ${ }^{1}$ \\ ${ }^{1}$ Chemical Engineering Department, Covenant University, Ota, Nigeria \\ ${ }^{2}$ Chemical Engineering Department, Obafemi Awolowo University, Ile-Ife, Nigeria \\ ${ }^{3}$ Mechanical Engineering Department, University of Ibadan, Ibadan, Nigeria \\ Email: temitayo.fatoki@covenantuniversity.edu.ng
}

Received 25 February 2015; accepted 22 June 2015; published 25 June 2015

Copyright (C) 2015 by authors and Scientific Research Publishing Inc.

This work is licensed under the Creative Commons Attribution International License (CC BY). http://creativecommons.org/licenses/by/4.0/

(c) (i) Open Access

\section{Abstract}

The dispersion models were used to estimate or predict the concentration of air pollutants or toxins emitted from sources such as industrial plants, vehicular traffic or accidental chemical releases. In this study, the Industrial Source Complex Short Term (ISCST3) emission dispersion model was used to measure the ground level concentration of criteria air pollutants within $50 \mathrm{~km}$ radius of location. This model considered emissions from major point sources of pollutants in four existing and twenty-three proposed Nigeria petroleum refineries. The obtained ground level concentration for 24-hr averaging periods of the criteria air pollutants at sensitive receptor around each of the refineries was compared with the National Ambient Air Quality Standards (NAAQS) of Nigeria, World Bank and World Health Organization (WHO) to determine their level of compliance. The highest ground level concentration predicted to be $450-1875 \mu \mathrm{g} / \mathrm{m}^{3}$ for $24-\mathrm{h}$ averaging period was obtained at Tonwei Oil Refinery, Ekeremor Local Government, Bayelsa State, while the lowest ground level concentration predicted to be $0.0099-0.1 \mu \mathrm{g} / \mathrm{m}^{3}$ for $24-\mathrm{h}$ averaging period was obtained at Amakpe International Refinery, Eket Local Government, Akwa Ibom State. Percentage set limits of criteria air pollutants ranging from $0.02 \%$ to $94.5 \%$ are within the set standard limits and no health risk is associated with areas around the plant's locations while percentage set limits of criteria pollutants ranging from 1.1 to 55.6 folds of the standards exceed the maximum permitted limits, hence affecting areas around the plants. The air quality standards guiding petroleum refinery emissions must be strictly considered, in order to ensure that the ground level concentrations do not exceed the required standard limits and prevent the adverse effects of air pollution in the Nigeria airshed.

\section{Keywords}

Air Quality Standards, Ground Level Concentration, Nigeria Petroleum Refinery 


\section{Introduction}

Each year industrial facilities discharge pollutants into the environment, releasing large amounts of pollutants leading to respiratory, neurological and reproductive disorders, and cancers [1]. Nigeria is presently a major emitter of criteria air emissions: carbon monoxide $(\mathrm{CO})$, oxides of nitrogen $\left(\mathrm{NO}_{\mathrm{x}}\right)$, particulate matter less than 10.0 microns in diameter $\left(\mathrm{PM}_{10}\right)$, sulphur dioxide $\left(\mathrm{SO}_{2}\right)$ and volatile organic compounds (VOCs) which comes from number of sources within a petroleum refinery including equipment leaks (from valves or other devices), hightemperature combustion processes, etc [2]. The Industrial Source Complex Short Term (ISCST3) air dispersion modeling tool is a steady state Gaussian plume model which can be used to assess pollutant concentrations, and/ or deposition fluxes from a wide variety of sources are associated with an industrial source complex [3]. It was used to predict the ground level concentrations of criteria air pollutants from the four major existing and twenty-three proposed petroleum refineries in Nigeria, within $50 \mathrm{~km}$ radius of location from point sources of emission in the refineries, for the prevailing meteorological conditions assuming a "worst case scenario" i.e. $100 \%$ operation rate. The model is used to determine whether existing or proposed new industrial facilities are or will be in compliance with the National Ambient Air Quality Standards (NAAQS). Air pollution models constitute a set of formulae that take into account sources of pollution in a given area, the amounts of pollutants emitted by each source, chemical reaction transformations, different meteorological conditions and topographical features, and other factors that affect dispersion of pollutants. [4] defined the process of computer modeling of air pollution as the ability to use computers to solve basic equations that described the dynamics of meteorological and air pollution phenomena, and ultimately, the adverse effects of air pollution. The basis for most of those models was the complete equation for Gaussian Dispersion Modeling of continuous and buoyant air pollution plumes [5]. Previous studies on investigation on emissions using ISCST3 include [5]-[7]. This study is carried out to investigate the atmospheric conditions of the petroleum refineries in Nigeria and identify the environmental impacts of emissions of criteria pollutants in the area of influence. Determinations of their ground level concentrations and comparison with the National Ambient Air Quality Standards (NAAQS) of Nigeria, World Bank and World Health Organization (WHO) will indicate the level of air quality management required around Nigerian petroleum refineries.

\section{Study Areas}

As shown in Figure 1, Nigeria has 4 existing refineries; Kaduna Refining and petrochemical company (KPRC) located at Chikun Local Government of Kaduna State operating at 110,000 bbl/day; Warri Refining and petrochemical company (WRPC) located at Warri southwest local government of Nigeria's Delta State with an installed capacity of 125,000 bbl/day; Port Harcourt Refining Company (PHRC) has two refineries at Alesa-Eleme, near Port Harcourt in Nigeria's Southern Province, Rivers State. Port Harcourt I refinery has installed capacity of $60,000 \mathrm{bbl} /$ day while Port Harcourt II refinery is the complex new refinery with installed capacity of 150,000 bbl/day [8] [9]. Due to scarcity of petroleum products, 23 proposed refineries have been licensed in Nigeria to ensure availability of the products (Figure 1), which includes the following with their installed capacities; Resources refinery and petrochemical Limited located in Ikot Abasi local government area of Akwa Ibom state (100,000 bbl/day); Amakpe International Refinery, Eket local government of Akwa Ibom state with production capacity of (12,000 bbl/day); Rehoboth Natural Resources Limited (12,000 bbl/day), Immingiri, Yenekoa local government of Bayelsa state; Amexum Corporation (100,000 bbl/day), Akpabuyo local government of Cross river state; Antonio Oil (27,000 bbl/day), Ipokia local government of Ogun state; Gasoline Associates International Limited Refinery (100,000 bbl/day), Ipokia local government of Ogun state; Ologbo Refinery Company Nigeria Limited (12,000 bbl/day), Ikpoba Okha local government of Edo state; Ode Aye Refinery Limted (100,000 bbl/day), Okiti pupa local government area of Ondo state; Orient Petroleum Resources Limited (55,000 bbl/day), Anambra West local government area of Anambra state; Qua Petroleum Refinery Limited (100,000 bbl/day), Ibeno local government of Akwa Ibom state; ChaseWood Consortium Nigeria Limited (70,000 bbl/day), Eket local government area of Akwa Ibom state; Clean Water Refinery (60,000 bbl/day), Eleme local government area of Rivers state; Niger Delta Refinery \& Petrochemical Limited (100,000 bbl/day), Warri-South local government area of Delta state; NSP Refineries and Oil Services Limited (120,000 bbl/day), Andoni local government area of Rivers state; RIVGAS Petroleum and Energy Limited (30,000 bbl/day), Port Harcourt local government area of Rivers state; Sapele Refinery Limited located in Okpe-Sobo, Sapele local government area of Delta state (100,000 bbl/day); Starrex Petroleum Refinery (100,000 bbl/day), Eleme local government area of 


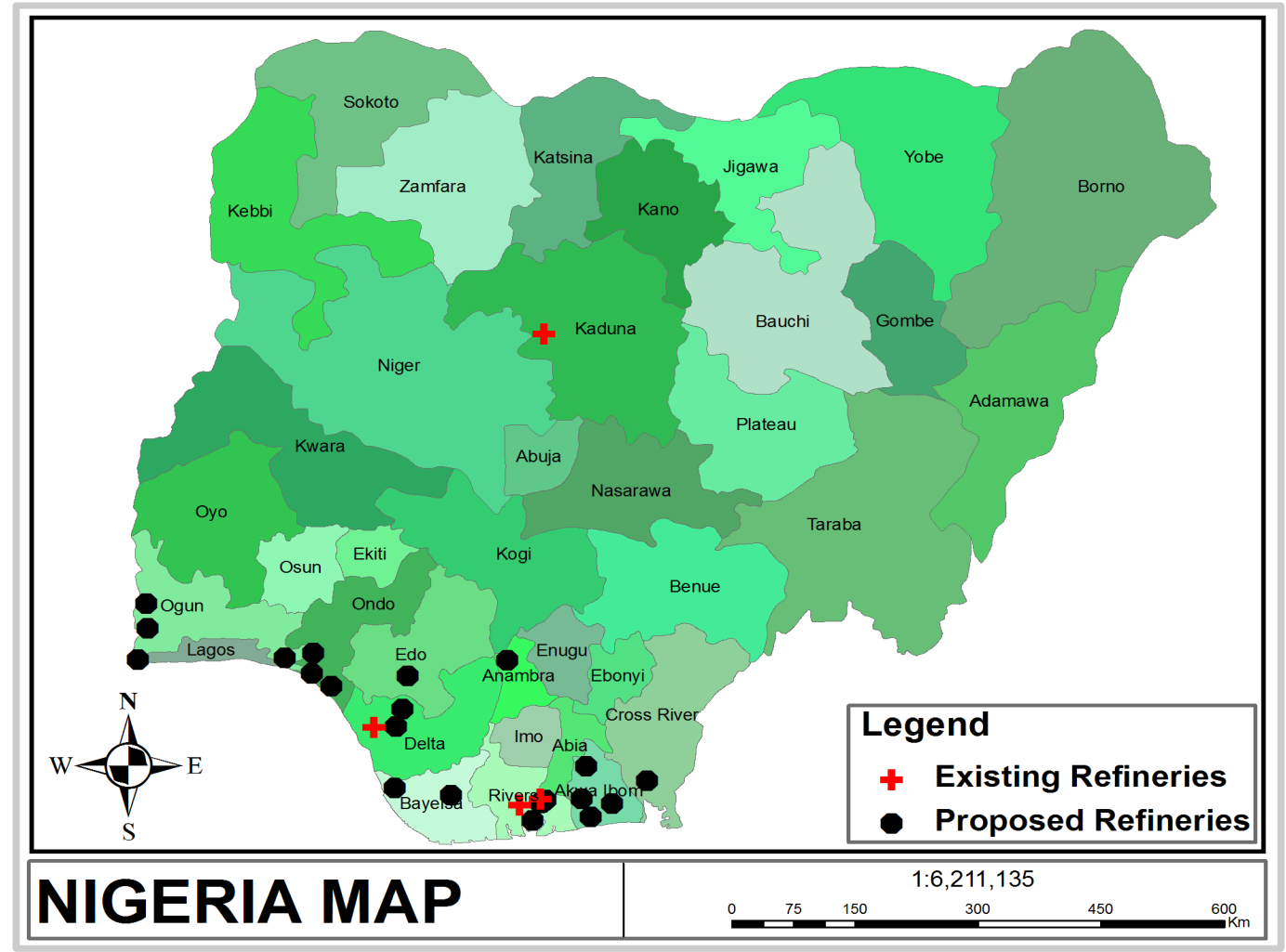

Figure 1. Map of Nigeria showing the existing and proposed Refineries.

Rivers state; Total Support Limited (12,000 bbl/day), Calabar Mnincipal local government area of Cross River state; Union Atlantic Petroleum Limited (100,000 bbl/day), Badagry local government area of Lagos state; Tonwei Oil Refinery (200,000 bbl/day), Ekeremor local government area of Bayelsa state; Ilaje Refinery and Petrochemicals (100,000 bbl/day), Ilaje local government area of Ondo state; Southwest Refinery and Petrochemical Company (100,000 bbl/day) is located in Ogun Waterside local government area of Ogun state; Owena Oil and gas Limited (100,000 bbl/day), Ilaje local government area of Ondo state [8]-[10].

\section{Methodology}

ISCST3 model uses steady state Gaussian plume equation to model emissions from point sources, including flares, process heaters, steam boilers and fluid catalytic cracking unit. This model considered emissions from the stacks of existing refineries and proposed petroleum refineries in Nigeria. Criteria air pollutants modeled for the ground level concentration include $\mathrm{CO}, \mathrm{NO}_{\mathrm{X}}, \mathrm{PM}_{10}, \mathrm{SO}_{2}$ and $\mathrm{VOC}$. The standards of ambient air quality as reported by NAAQS,WB and WHO is shown in Table 1. Major sources of emissions and parameters used as input to the air dispersion modeling for each of the refineries are shown in Table 2 and Table 3. Some proposed refineries have similar operation rate, hence will have the same emission sources and dispersion model parameter. The ISC-AERMOD View air dispersion modeling tool developed specially for Microsoft Windows and runs under Windows 95/98/Me/NT/2000 and XP was employed in this study. This is a complete and powerful air dispersion modeling package which incorporates the popular U.S. EPA models into one interface: AERMOD, ISCST3, and ISC-PRIME. ISCST3 dispersion model interfaces [1]. The ISCST3 interface was used in this study which uses pathways that compose the run stream file as the basics for its functional organization. These pathways include:

i) Control Pathway (CO): Where the modeling scenario (worst case scenario i.e. 100\% operation rate), the pollutants $\left(\mathrm{CO}, \mathrm{NO}_{\mathrm{X}}, \mathrm{SO}_{2}, \mathrm{VOC}\right.$ and $\left.\mathrm{PM}\right)$ and the averaging periods (24-hr) are specified.

ii) Source Pathway (SO): Where the sources of pollutant emissions (point sources of pollutants in the refinery-process heaters, fluid catalytic cracking units, steam boilers and flares) are defined. 
Table 1. Standards of ambient air quality.

\begin{tabular}{cccccc}
\hline & & & \multicolumn{3}{c}{ Maximum Concentration $\left(\mu \mathrm{g} / \mathrm{m}^{3}\right)$} \\
\cline { 4 - 5 } & Pollutants & Averaging Period & NAAQS & World Bank & WHO \\
\hline 1 & $\mathrm{CO}$ & $24-\mathrm{Hr}$ & 11,400 & - & - \\
2 & $\mathrm{NO}_{\mathrm{x}}$ & $24-\mathrm{Hr}$ & 75 & 150 & 20 \\
3 & $\mathrm{SO}_{2}$ & $24-\mathrm{Hr}$ & 26 & - & 50 \\
4 & $\mathrm{PM}$ & $24-\mathrm{Hr}$ & 250 & - & - \\
5
\end{tabular}

Source: FEPA (1991) [11]; World Bank (1998) [12]; WHO (2005) [13].

Table 2. Emission sources and dispersion model parameters for existing refineries.

\begin{tabular}{|c|c|c|c|c|c|c|c|c|c|c|}
\hline \multirow{2}{*}{ Plant Name } & \multirow{2}{*}{ Stack } & \multirow{2}{*}{$\begin{array}{l}\text { Discharge } \\
\text { Temperature } \\
\text { (k) }\end{array}$} & \multirow{2}{*}{$\begin{array}{c}\text { Stack } \\
\text { Diameter } \\
(\mathrm{m})\end{array}$} & \multirow{2}{*}{$\begin{array}{c}\text { Exit } \\
\text { Velocity } \\
(\mathrm{m} / \mathrm{s})\end{array}$} & \multirow{2}{*}{$\begin{array}{c}\text { Release } \\
\text { Height (m) }\end{array}$} & \multicolumn{5}{|c|}{${ }^{\mathrm{c}}$ Emission $(\mathrm{g} / \mathrm{s})$} \\
\hline & & & & & & $\mathrm{PM}_{10}$ & $\mathrm{SO}_{2}$ & $\mathrm{NO}_{\mathrm{x}}$ & VOC & $\mathrm{CO}$ \\
\hline \multirow{9}{*}{$\begin{array}{c}\text { Kaduna } \\
\text { Refining \& } \\
\text { Petrochemical } \\
\text { Company (KRPC) } \\
110,000 \text { BPSD }\end{array}$} & ${ }^{\mathrm{a}}$ Flare 1 & 1540 & 6.6 & 4.52 & 66 & - & - & 0.001 & 0.003 & 0.005 \\
\hline & Flare 2 & 1540 & 6.6 & 4.52 & 66 & - & - & 0.001 & 0.003 & 0.005 \\
\hline & Flare 3 & 1540 & 6.6 & 4.52 & 66 & - & - & 0.001 & 0.003 & 0.005 \\
\hline & ${ }^{b} \mathrm{FCCU}$ & 330 & 2.75 & 23.47 & 67 & - & 54.5 & 7.9 & 24.3 & 1510.4 \\
\hline & ${ }^{\mathrm{b}}$ Boiler 1 & 550 & 2.18 & 9.05 & 30 & - & 304.1 & 1420.1 & - & 106 \\
\hline & Boiler 2 & 550 & 2.18 & 9.05 & 30 & - & 304.1 & 1420.1 & - & 106 \\
\hline & Boiler 3 & 550 & 2.18 & 9.05 & 30 & - & 304.1 & 1420.1 & - & 106 \\
\hline & ${ }^{\mathrm{b}}$ Heater 1 & 660 & 2.01 & 8.03 & 43 & 2.1 & 45.1 & 173.2 & 1.0 & 15.8 \\
\hline & Heater 2 & 660 & 2.01 & 8.03 & 43 & 2.1 & 45.1 & 173.2 & 1.0 & 15.8 \\
\hline \multirow{7}{*}{$\begin{array}{c}\text { Port Harcourt } \\
\text { Refining Company } \\
\text { I } \\
\text { (PHRC) } \\
60,000 \text { BPSD }\end{array}$} & ${ }^{\mathrm{a}}$ Flare 1 & 840 & 1.1 & 2.47 & 36 & - & - & 0.0003 & 0.0006 & 0.002 \\
\hline & Flare 2 & 840 & 1.1 & 2.47 & 36 & - & - & 0.0003 & 0.0006 & 0.002 \\
\hline & ${ }^{\mathrm{b}} \mathrm{FCCU}$ & 180 & 1.5 & 12.8 & 37 & - & 12.5 & 2.0 & 5.6 & 347.7 \\
\hline & ${ }^{b}$ Boiler 1 & 300 & 1.19 & 4.94 & 16 & - & 59.6 & 278.7 & - & 20.8 \\
\hline & Boiler 2 & 300 & 1.19 & 4.94 & 16 & - & 59.6 & 278.7 & - & 20.8 \\
\hline & ${ }^{\mathrm{b}}$ Heater 1 & 360 & 1.1 & 4.38 & 24 & 0.6 & 13.4 & 51.6 & 0.3 & 4.7 \\
\hline & Heater 2 & 360 & 1.1 & 4.38 & 24 & 0.6 & 13.4 & 51.6 & 0.3 & 4.7 \\
\hline \multirow{11}{*}{$\begin{array}{l}\text { Port Harcourt } \\
\text { Refining } \\
\text { Company II } \\
\text { (PHRC) } \\
\text { 150,000 BPSD }\end{array}$} & a Flare 1 & 2100 & 2.75 & 6.17 & 90 & - & - & 0.002 & 0.004 & 0.01 \\
\hline & Flare 2 & 2100 & 2.75 & 6.17 & 90 & - & - & 0.002 & 0.004 & 0.01 \\
\hline & Flare 3 & 2100 & 2.75 & 6.17 & 90 & - & - & 0.002 & 0.004 & 0.01 \\
\hline & Flare 4 & 2100 & 2.75 & 6.17 & 90 & & & 0.002 & 0.004 & 0.01 \\
\hline & ${ }^{\mathrm{b}} \mathrm{FCCU}$ & 450 & 3.75 & 32.01 & 92 & - & 103.5 & 14.9 & 46.3 & 2877 \\
\hline & ${ }^{b}$ Boiler 1 & 750 & 2.97 & 12.35 & 41 & - & 267.8 & 1250.7 & - & 93.4 \\
\hline & Boiler 2 & 750 & 2.97 & 12.35 & 41 & - & 267.8 & 1250.7 & - & 93.4 \\
\hline & ${ }^{b}$ Boiler 1 & 750 & 2.97 & 12.35 & 41 & - & 267.8 & 1250.7 & - & 93.4 \\
\hline & Boiler 2 & 750 & 2.97 & 12.35 & 41 & - & 267.8 & 1250.7 & - & 93.4 \\
\hline & ${ }^{\mathrm{b}}$ Heater 1 & 900 & 2.75 & 10.95 & 59 & 3.7 & 80.2 & 308.1 & 1.7 & 28 \\
\hline & Heater 2 & 900 & 2.75 & 10.95 & 59 & 3.7 & 80.2 & 308.1 & 1.7 & 28 \\
\hline
\end{tabular}




\section{Continued}

\begin{tabular}{ccccccccccc}
\hline & ${ }^{\mathrm{a}}$ Flare 1 & 1750 & 2.29 & 5.13 & 75 & - & - & 0.001 & 0.003 & 0.007 \\
& Flare 2 & 1750 & 2.29 & 5.13 & 75 & - & - & 0.001 & 0.003 & 0.007 \\
& Flare 3 & 1750 & 2.29 & 5.13 & 75 & - & - & 0.001 & 0.003 & 0.007 \\
$\begin{array}{c}\text { Warri } \\
\text { Refining \& }\end{array}$ & Flare 4 & 1750 & 2.29 & 5.13 & 75 & & & 0.001 & 0.003 & 0.007 \\
$\begin{array}{c}\text { Petrochemical } \\
\text { Company (WRPC) }\end{array}$ & ${ }^{\mathrm{b}}$ FCCU & 375 & 3.13 & 26.68 & 76 & - & 67.3 & 9.7 & 30 & 1870.1 \\
125,000 BPSD & Boiler 1 & 625 & 2.48 & 10.29 & 34 & - & 228.8 & 1068.3 & - & 79.8 \\
& Boiler 2 & 625 & 2.48 & 10.29 & 34 & - & 228.8 & 1068.3 & - & 79.8 \\
& Boiler 3 & 625 & 2.48 & 10.29 & 34 & & 228.8 & 1068.3 & - & 79.8 \\
& ${ }^{\mathrm{b}}$ Heater 1 & 750 & 2.29 & 9.13 & 49 & 2.9 & 61.3 & 253.3 & 1.3 & 21.4 \\
& Heater 2 & 750 & 2.29 & 9.13 & 49 & 2.9 & 61.3 & 253.3 & 1.3 & 21.4 \\
\hline
\end{tabular}

Source: a Abdul-Wahab et al. (2001) [14]; ' Stell (2000) [15]; 'CSEPA 1989 [16].

Table 3. Emission sources and dispersion model parameters for proposed refineries.

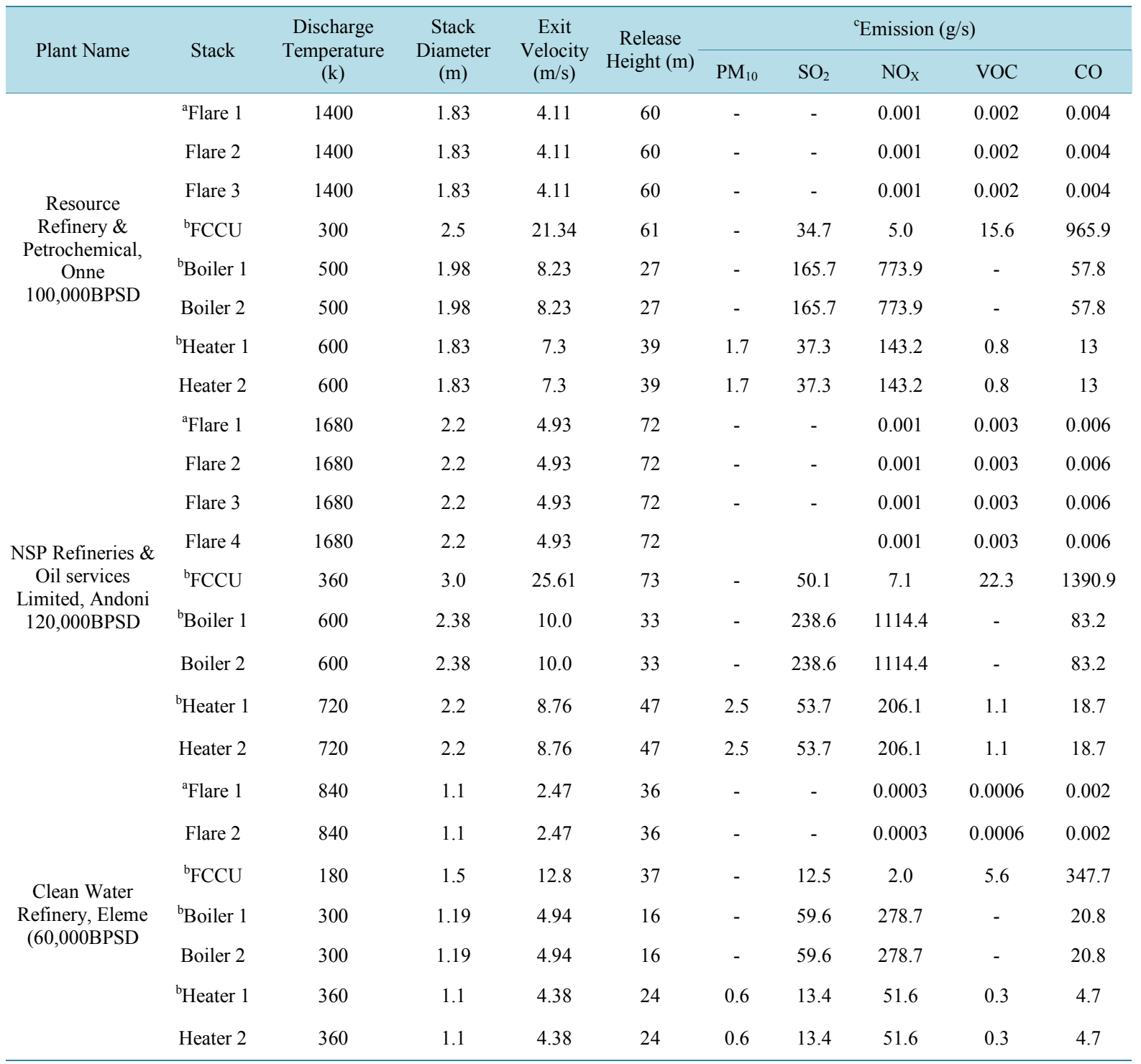




\section{Continued}

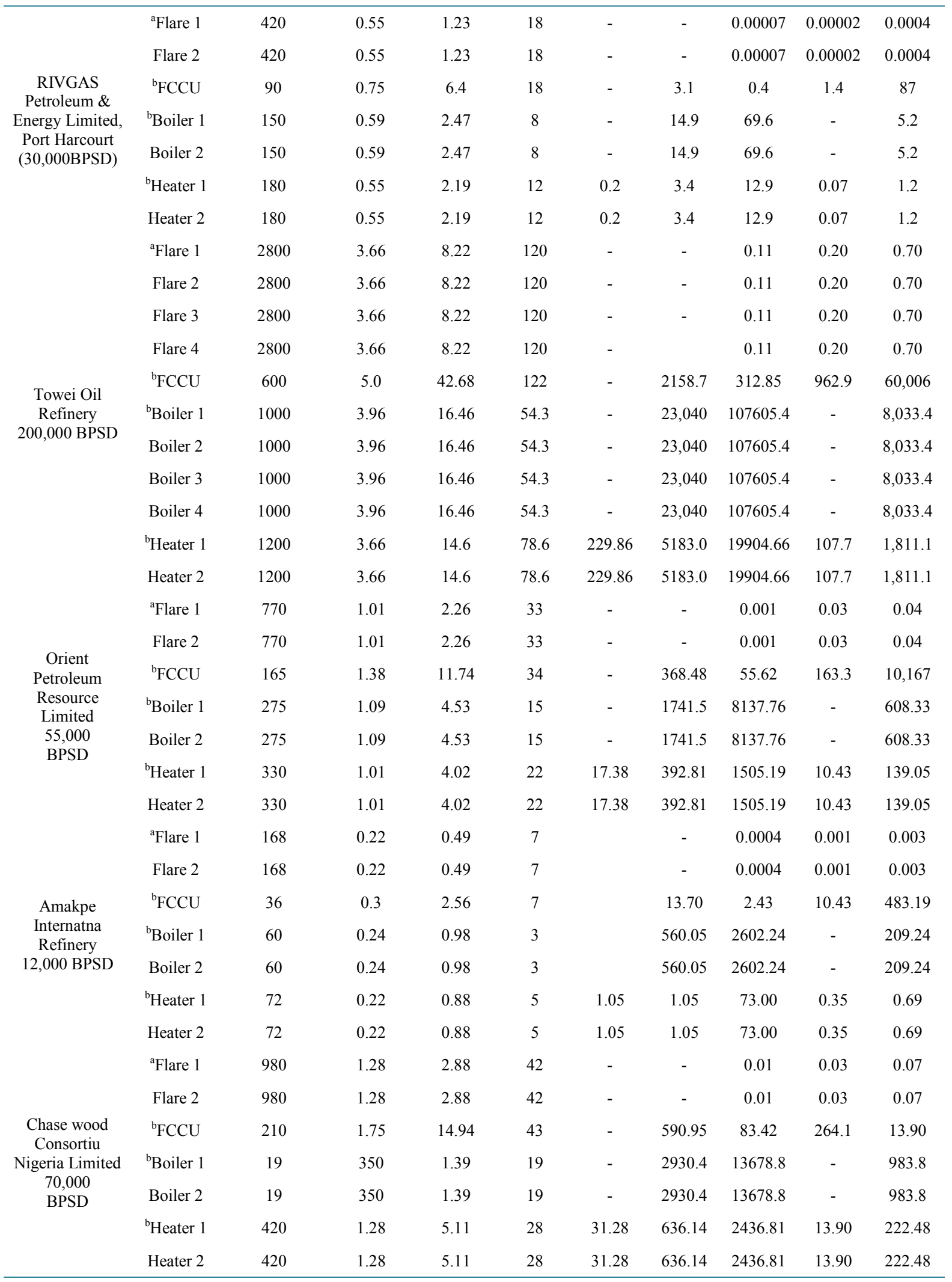

Source: a Abdul-Wahab et al. (2001); 'btell (2000); 'USEPA (1989). 
iii) Receptor Pathway (RE): where the receptors which determine the air quality impact at specific locations. Specifically, $50 \mathrm{~km}$ radius within each refinery was given adequate attention.

iv) Meteorological Pathway (ME): Where the atmospheric condition of the areas being modeled is defined, so it can be taken into account when determining the distribution of air pollution impacts for the area. Due to lack of upper air observations in Nigeria airport, meteorological database for the project location was combined using data from Cotonou meteorological observations. Cotonou has winds having prevalence for a southwesterly direction which is consistent with the winds observed in the refineries.

v) Terrain Grid Pathway (TG): Where the option of gridded terrain data to be used in calculating dry depletion in elevated or complex terrain is specified. Elevated terrain is relevant to this study.

(vi) Output Pathway (OU): Where you define which output results are necessary to meet the needs of the air quality modeling analysis. The resultant output is the ground level concentration of pollutants as shown in Figure 2 and Figure 3.

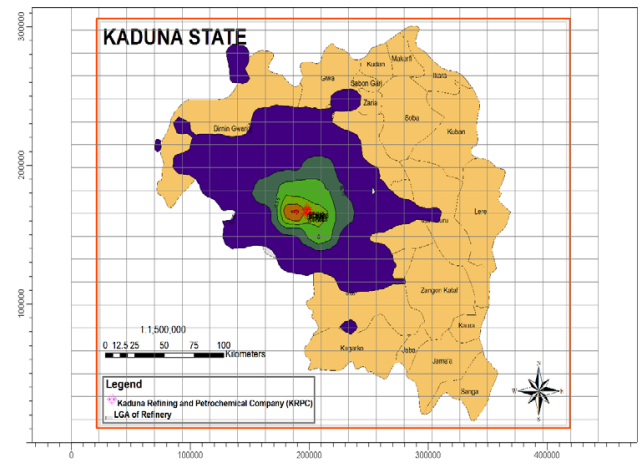

(a)

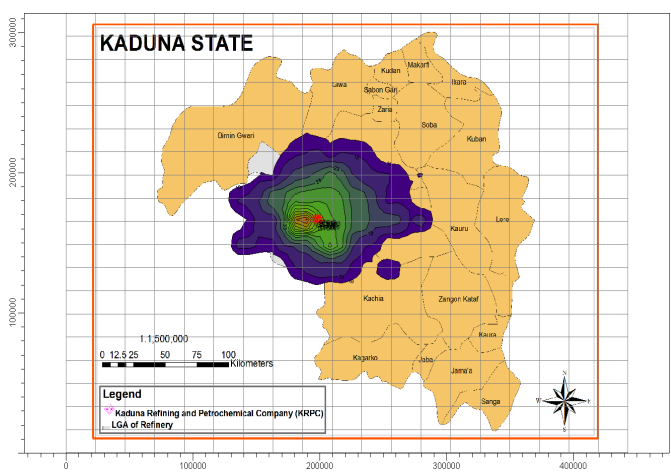

(c)

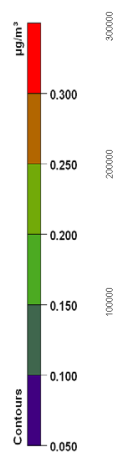

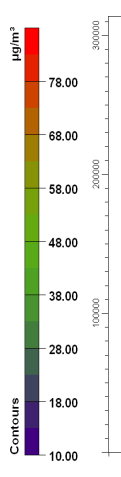

.

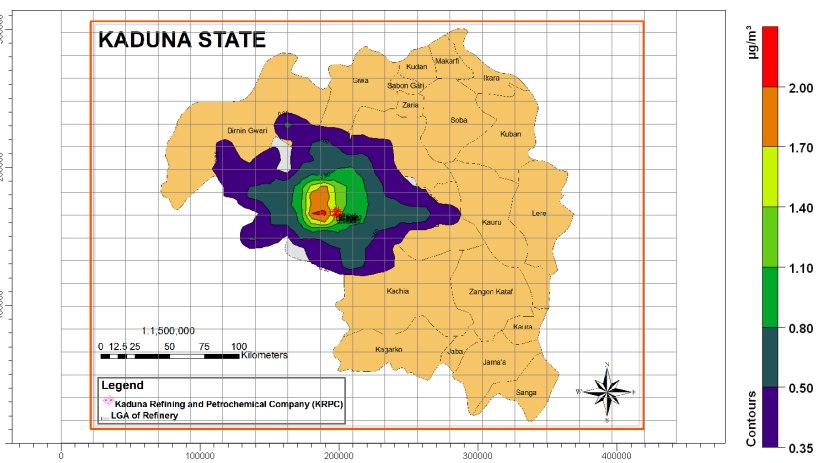

(b)

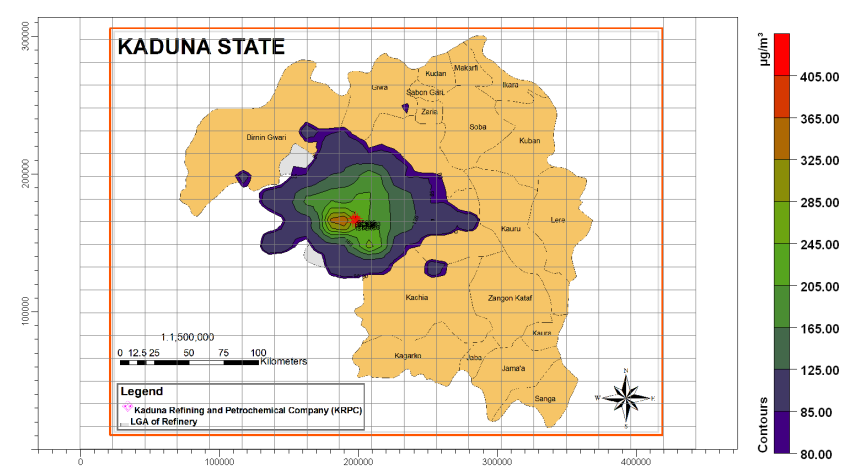

(e)

Figure 2. Predicted 24-hr Ground Level Concentrations of Criteria Pollutants from Kaduna Refining and Petrochemical Company, Kaduna State. (a) Predicted 24-hr PM 10 Concentrations; (b) Predicted 24-hr VOC Concentrations; (c) Predicted 24-hr SO 2 Concentrations; (d) Predicted 24-hr NO Concentrations; (e) Predicted 24-hr CO Concentrations. 


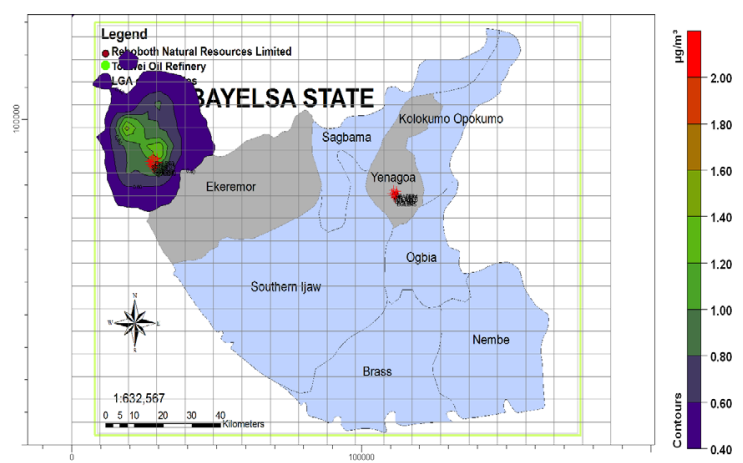

(a)

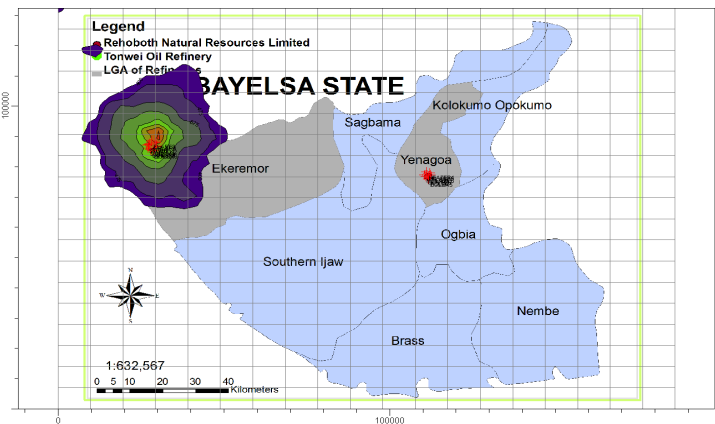

(c)

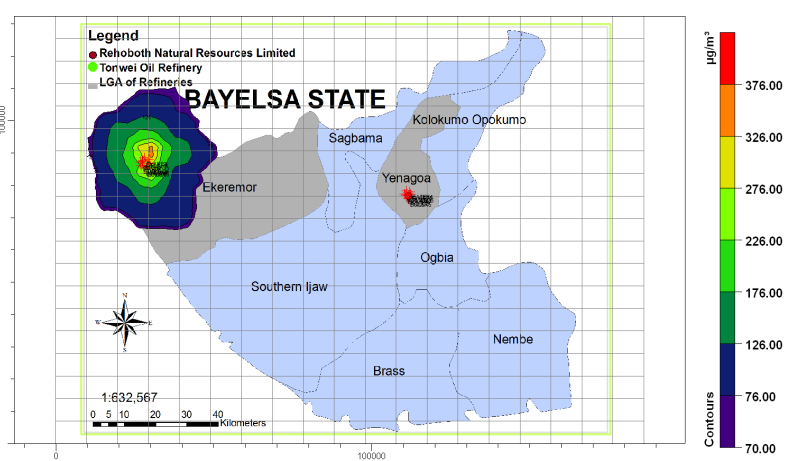

(b)
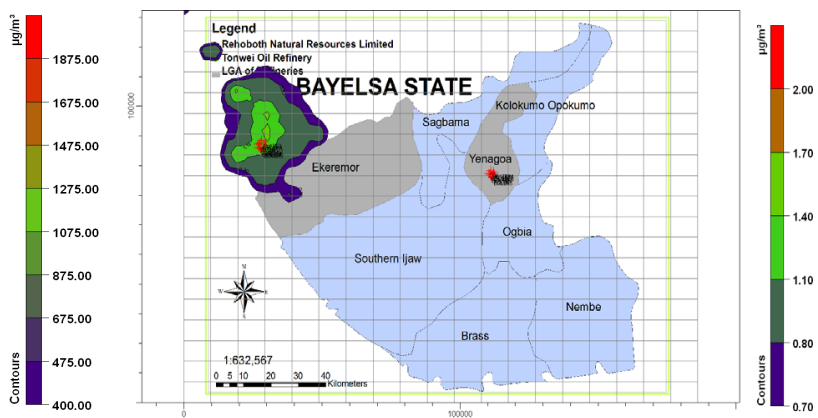

(d)

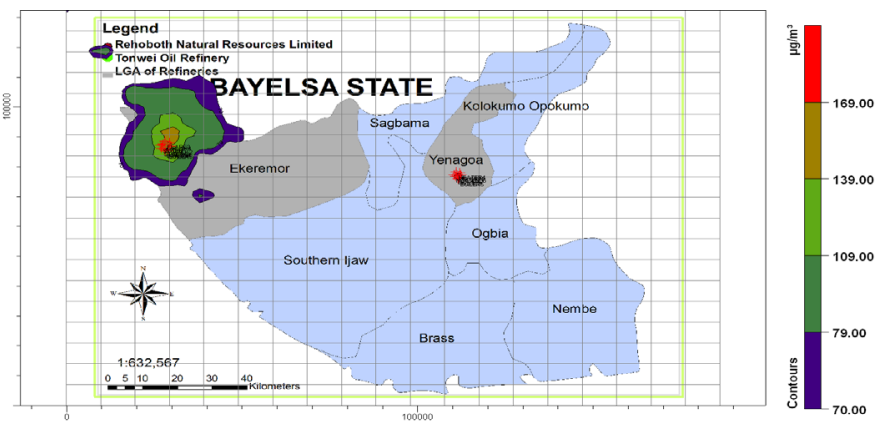

(e)

Figure 3. Cumulative 24-hr Ground Level Concentrations of Criteria Pollutants from Bayelsa state refineries. (a): Cumulative 24-hr PM $\mathrm{PM}_{10}$ Concentrations; (b) Cumulative 24-hr SO${ }_{2}$ Concentrations; (c) Cumulative 24-hr $\mathrm{NO}_{\mathrm{X}}$ Concentrations; (d) Cumulative 24-hr VOC Concentrations; (e) Cumulative 24-hr SO 2 Concentrations.

From the existing and proposed refineries, the ground level concentration (glc) of the criteria air pollutant were computed and compared with the standards of ambient air quality derived from National Ambient Air Quality Standards (NAAQS) of Federal Ministry of Environment (FMENV), that of the World Bank environmental guidelines and World Health Organization (WHO) standards to determine their level of compliance (Table 4 and Table 5).

\section{Results and Discussion}

An air quality impact on refineries is important to assess the impacts of emissions from petroleum refinery operations on their environment. Table 2 and Table 3 shows the emission sources and dispersion model parameters for the existing and proposed refineries these include the emission estimate of criteria pollutants from point sources (FCCU, Process heaters, Flares, Steam boilers) of emissions from the refineries. The dispersion modeling outputs from the ISC-AERMOD View (for the existing and proposed refineries in Nigeria) for 1-hour, 8hour, 24-hour and annual averaging periods for the criteria pollutants are shown in Figure 2 and Figure 3 
Table 4. 24-hr averaging period maximum ground level concentration of criteria pollutants and \% set limits in existing refineries.

\begin{tabular}{|c|c|c|c|c|c|}
\hline $\mathrm{S} / \mathrm{N}$ & Refinery & Air Pollutant & $\begin{array}{l}\text { Maximum glc } \\
\left(\mu \mathrm{g} / \mathrm{m}^{3}\right)\end{array}$ & $\begin{array}{l}\text { Maximum Impact Local } \\
\text { Government Area }\end{array}$ & $\begin{array}{c}\text { \% Set Limit of FMENV, } \\
\text { WB, WHO }\end{array}$ \\
\hline \multirow{5}{*}{1} & \multirow{5}{*}{$\begin{array}{c}\text { Kaduna } \\
\text { Refining \& } \\
\text { Petrochemical } \\
\text { Company (KRPC) } \\
110,000 \text { BPSD }\end{array}$} & $\mathrm{PM}_{10}$ & 0.4 & Chikun & 0.2 \\
\hline & & $\mathrm{SO}_{2}$ & 82.7 & Chikun & $3.2^{\mathrm{a}}$ \\
\hline & & $\mathrm{NO}_{\mathrm{x}}$ & 404.9 & Chikun & $5.4^{\mathrm{a}}$ \\
\hline & & $\mathrm{CO}$ & 164.1 & Chikun & 1.4 \\
\hline & & VOC & $2 . .3$ & Chikun & 0.04 \\
\hline \multirow{5}{*}{2} & \multirow{5}{*}{$\begin{array}{c}\text { Port Harcourt Refining } \\
\text { Company I } \\
\text { (PHRC) } \\
60,000 \text { BPSD }\end{array}$} & $\mathrm{PM}_{10}$ & 0.2 & Eleme & 0.08 \\
\hline & & $\mathrm{SO}_{2}$ & 26.3 & Eleme & $1.02^{\mathrm{a}}$ \\
\hline & & $\mathrm{NO}_{\mathrm{x}}$ & 121.4 & Eleme & $1.6^{\mathrm{a}}$ \\
\hline & & $\mathrm{CO}$ & 73.0 & Eleme & 0.6 \\
\hline & & VOC & 1.0 & Eleme & 0.02 \\
\hline \multirow{5}{*}{3} & \multirow{5}{*}{$\begin{array}{c}\text { Port Harcourt } \\
\text { Refining Company II } \\
\text { (PHRC) } \\
\text { 150,000 BPS }\end{array}$} & $\mathrm{PM}_{10}$ & 0.7 & Eleme & 0.3 \\
\hline & & $\mathrm{SO}_{2}$ & 111.9 & Eleme & $4.3^{\mathrm{a}}$ \\
\hline & & $\mathrm{NO}_{\mathrm{x}}$ & $3,589.1$ & Eleme & $4.7^{\mathrm{a}}$ \\
\hline & & $\mathrm{CO}$ & 214.9 & Eleme & 1.9 \\
\hline & & VOC & 17.6 & Eleme & 0.3 \\
\hline \multirow{5}{*}{4} & & $\mathrm{PM}_{10}$ & 1.7 & Warri S/W & 0.7 \\
\hline & $\begin{array}{c}\text { Warri } \\
\text { Refining \& }\end{array}$ & $\mathrm{SO}_{2}$ & 444.4 & Warri S/W & $1.7^{\mathrm{a}}$ \\
\hline & $\begin{array}{c}\text { Petrochemical } \\
\text { Company (WRPC) }\end{array}$ & $\mathrm{NO}_{\mathrm{x}}$ & $2,145.8$ & Warri S/W & $2.9^{\mathrm{a}}$ \\
\hline & 125,000 BPSD & $\mathrm{CO}$ & 479.2 & Warri S/W & 4.2 \\
\hline & & VOC & 0.9 & Warri S/W & 0.1 \\
\hline
\end{tabular}

${ }^{\text {a }}$ folds of $\%$ set limits of the standard i.e. above the set standard limits.

(surface plots available on request) while the Tables showing the results of maximum ground Level Concentration of air pollutant within $50 \mathrm{~km}$ from each of the refineries are shown in Table 4 and the maximum ground level concentration impact from $50 \mathrm{~km}$ receptor locations obtained when the ground level concentration of the criteria pollutants from the refineries was compared with the set limits of Nigeria's National Ambient Air Quality Standard (NAAQS), World Bank and World Health Organization (WHO) are shown in Table 5 and Table 6. The results predicted the total emissions of criteria pollutant emitted per annum in the existing and proposed refineries where $\mathrm{NO}_{x}$ has the highest rate of emissions. Also, from the existing refineries, Port Harcourt 2 refinery has the highest criteria pollutant emission rate due to its high installed capacity $(150,000 \mathrm{bpsd})$ while from the proposed refineries Tonwei refinery has the highest rate of emissions of the criteria pollutant due to its high installed capacity $(200,000 \mathrm{bpsd})$. It was observed that some refineries exceeds their area of influence i.e. the emission dispersion exceeds the plant location to the neighbouring local government area e.g. $\mathrm{CO}$ emissions for Rehoboth for $1 \mathrm{~h}$ averaging periods dispersed from Yenegoa to Ogbia. From Table 5 and Table 6, criteria pollutants of ranges $0.02 \%-94.5 \%$ of the standards falls within the set standards limit which means there is no health risk in the receptor locations while criteria pollutants of ranges $1.1-55.6$ folds of the standards have exceeded the set standard limits and it means there is health risk in the receptor locations.

\section{Conclusions}

The ISCST3 dispersion model has been used in this study to evaluate the ground level concentrations from the 
Table 5. 24-hr averaging period maximum ground level concentration of criteria pollutants and \% set limits in proposed refineries.

\begin{tabular}{|c|c|c|c|c|c|}
\hline $\mathrm{S} / \mathrm{N}$ & Refinery & Air Pollutant & $\begin{array}{l}\text { Maximum glc } \\
\left(\mu \mathrm{g} / \mathrm{m}^{3}\right)\end{array}$ & $\begin{array}{l}\text { Maximum Impact Local } \\
\text { Government Area }\end{array}$ & $\begin{array}{c}\text { \% Set Limit of FMENV, } \\
\text { WB, WHO }\end{array}$ \\
\hline \multirow{5}{*}{1} & \multirow{5}{*}{$\begin{array}{l}\text { Amakpe International } \\
\text { Refinery } \\
(12,000 \text { bpsd })\end{array}$} & $\mathrm{PM}_{10}$ & 0.04 & Ikot Ekpene & 0.02 \\
\hline & & $\mathrm{SO}_{2}$ & 4.4 & Ikot Ekpene & 16.9 \\
\hline & & $\mathrm{NO}_{\mathrm{x}}$ & 22.6 & Ikot Ekpene & 30.1 \\
\hline & & $\mathrm{CO}$ & 10.7 & Ikot Ekpene & 0.04 \\
\hline & & VOC & 0.2 & Ikot Ekpene & 0.002 \\
\hline \multirow{5}{*}{2} & \multirow{5}{*}{$\begin{array}{l}\text { Amexum Corporation } \\
\quad(100,000 \text { bpsd })\end{array}$} & $\mathrm{PM}_{10}$ & 0.5 & Akpabuyo & 0.2 \\
\hline & & $\mathrm{SO}_{2}$ & 6.7 & Akpabuyo & 0.4 \\
\hline & & $\mathrm{NO}_{\mathrm{x}}$ & 314 & Akpabuyo & $0.4^{\mathrm{a}}$ \\
\hline & & $\mathrm{CO}$ & 215 & Akpabuyo & 1.9 \\
\hline & & VOC & 4.1 & Akpabuyo & 0.1 \\
\hline \multirow{5}{*}{3} & \multirow{5}{*}{$\begin{array}{c}\text { Antonio Oil } \\
\text { (27,000 bpsd) }\end{array}$} & $\mathrm{PM}_{10}$ & 0.5 & Ipokia & 0.2 \\
\hline & & $\mathrm{SO}_{2}$ & 99.4 & Ipokia & $3.8^{\mathrm{a}}$ \\
\hline & & $\mathrm{NO}_{\mathrm{x}}$ & 413 & Ipokia & 1.5 \\
\hline & & $\mathrm{CO}$ & 37.5 & Ipokia & 0.3 \\
\hline & & VOC & 1.8 & Ipokia & 0.03 \\
\hline \multirow{5}{*}{4} & \multirow{5}{*}{$\begin{array}{l}\text { Chase Wood Consortium } \\
\text { Nigeria Limited } \\
(70,000 \text { bpsd })\end{array}$} & $\mathrm{PM}_{10}$ & 0.5 & Eket & 0.2 \\
\hline & & $\mathrm{SO}_{2}$ & 210 & Eket & $0.8^{\mathrm{a}}$ \\
\hline & & $\mathrm{NO}_{\mathrm{x}}$ & 1500 & Eket & $2.0^{\mathrm{a}}$ \\
\hline & & $\mathrm{CO}$ & 170 & Eket & 0.01 \\
\hline & & VOC & 26 & Eket & 0.4 \\
\hline \multirow{5}{*}{5} & \multirow{5}{*}{$\begin{array}{l}\text { Clean Water Refinery } \\
(60,000 \text { bpsd })\end{array}$} & $\mathrm{PM}_{10}$ & 0.5 & Eleme & 0.2 \\
\hline & & $\mathrm{SO}_{2}$ & 39.5 & Eleme & $1.3^{\mathrm{a}}$ \\
\hline & & $\mathrm{NO}_{\mathrm{x}}$ & 185.5 & Eleme & $2.0^{\mathrm{a}}$ \\
\hline & & $\mathrm{CO}$ & 102.4 & Eleme & 0.9 \\
\hline & & VOC & 1.6 & Eleme & 0.02 \\
\hline \multirow{5}{*}{6} & \multirow{5}{*}{$\begin{array}{c}\text { Gasoline Associate \& } \\
\text { International Limited } \\
\text { Refinery } \\
(100,000 \text { bpsd }\end{array}$} & $\mathrm{PM}_{10}$ & 0.5 & Ipokia & 0.2 \\
\hline & & $\mathrm{SO}_{2}$ & 58.6 & Ipokia & $2.3^{\mathrm{a}}$ \\
\hline & & $\mathrm{NO}_{\mathrm{x}}$ & 281.5 & Ipokia & $3.8^{\mathrm{a}}$ \\
\hline & & $\mathrm{CO}$ & 139.8 & Ipokia & 1.2 \\
\hline & & VOC & 2.1 & Ipokia & 0.04 \\
\hline \multirow{5}{*}{7} & \multirow{5}{*}{$\begin{array}{l}\text { Ilaje Refinery \& } \\
\text { Petrochemicals } \\
(100,000 \text { bpsd })\end{array}$} & $\mathrm{PM}_{10}$ & 0.8 & Ilaje & 0.3 \\
\hline & & $\mathrm{SO}_{2}$ & 61 & Ilaje & $0.2^{\mathrm{a}}$ \\
\hline & & $\mathrm{NO}_{\mathrm{x}}$ & 298 & Ilaje & $3.9^{\mathrm{a}}$ \\
\hline & & $\mathrm{CO}$ & 192 & Ilaje & 1,7 \\
\hline & & VOC & 3.0 & Ilaje & 0.05 \\
\hline
\end{tabular}




\section{Continued}

\begin{tabular}{|c|c|c|c|c|c|}
\hline \multirow{5}{*}{8} & \multirow{5}{*}{$\begin{array}{l}\text { Niger Delta Refinery \& } \\
\text { Petrochemical Limited } \\
\quad(100,000 \text { bpsd })\end{array}$} & $\mathrm{PM}_{10}$ & 0.5 & Warri South & 0.2 \\
\hline & & $\mathrm{SO}_{2}$ & 72.7 & Warri South & $2.8^{\mathrm{a}}$ \\
\hline & & $\mathrm{NO}_{\mathrm{x}}$ & 347.3 & Warri South & $4.6^{\mathrm{a}}$ \\
\hline & & $\mathrm{CO}$ & 417.1 & Warri South & 3.7 \\
\hline & & VOC & 6.7 & Warri South & 0.1 \\
\hline \multirow{5}{*}{9} & \multirow{5}{*}{$\begin{array}{l}\text { NSP Refineries and Oil } \\
\text { Services Limited } \\
\text { (120,000 bpsd) }\end{array}$} & $\mathrm{PM}_{10}$ & 0.9 & Andoni & 0.4 \\
\hline & & $\mathrm{SO}_{2}$ & 395.0 & Andoni & $1.5^{\mathrm{a}}$ \\
\hline & & $\mathrm{NO}_{\mathrm{x}}$ & 1903.0 & Andoni & $1.3^{\mathrm{a}}$ \\
\hline & & $\mathrm{CO}$ & 375.0 & Andoni & 3.3 \\
\hline & & VOC & 6.0 & Andoni & 0.1 \\
\hline \multirow{5}{*}{10} & \multirow{6}{*}{$\begin{array}{l}\text { Ode Aye Refinery Limited } \\
(100,000 \text { bpsd })\end{array}$} & $\mathrm{PM}_{10}$ & 0.4 & Okiti pupa & 0.2 \\
\hline & & $\mathrm{SO}_{2}$ & 83.5 & Okiti pupa & $32.1^{\mathrm{a}}$ \\
\hline & & $\mathrm{NO}_{\mathrm{x}}$ & 440 & Okiti pupa & $5.9^{\mathrm{a}}$ \\
\hline & & $\mathrm{CO}$ & 113.8 & Okiti pupa & 1.0 \\
\hline & & VOC & 2.0 & Okiti pupa & 0.03 \\
\hline \multirow{5}{*}{11} & & $\mathrm{PM}_{10}$ & 0.05 & Ikpoba-oha & 0.02 \\
\hline & \multirow{4}{*}{$\begin{array}{c}\text { Ologbo Refinery } \\
\text { Company Nigeria Limited } \\
(12,000 \text { bpsd })\end{array}$} & $\mathrm{SO}_{2}$ & 0.9 & Ikpoba-oha & $3.5^{\mathrm{a}}$ \\
\hline & & $\mathrm{NO}_{\mathrm{x}}$ & 8.9 & Ikpoba-oha & 11.8 \\
\hline & & $\mathrm{CO}$ & 5.0 & Ikpoba-oha & 0.04 \\
\hline & & VOC & 0.1 & Ikpoba-oha & 0.002 \\
\hline \multirow{5}{*}{12} & \multirow{5}{*}{$\begin{array}{l}\text { Orient Petroleum Resources } \\
\text { Limited }(55,000 \text { bpsd) }\end{array}$} & $\mathrm{PM}_{10}$ & 0.7 & Anambra West & 0.3 \\
\hline & & $\mathrm{SO}_{2}$ & 0.9 & Anambra West & 3.5 \\
\hline & & $\mathrm{NO}_{\mathrm{x}}$ & 8.5 & Anambra West & 11.3 \\
\hline & & $\mathrm{CO}$ & 219.6 & Anambra West & 1.9 \\
\hline & & VOC & 3.0 & Anambra West & 0.01 \\
\hline \multirow{5}{*}{13} & \multirow{5}{*}{$\begin{array}{l}\text { Owena Oil \& Gas } \\
\text { Limited } \\
(60,000 \text { bpsd })\end{array}$} & $\mathrm{PM}_{10}$ & 0.2 & Ilaje & 0.08 \\
\hline & & $\mathrm{SO}_{2}$ & 34.5 & Ilaje & $13.3^{\mathrm{a}}$ \\
\hline & & $\mathrm{NO}_{\mathrm{x}}$ & 159.4 & Ilaje & $2.2^{\mathrm{a}}$ \\
\hline & & $\mathrm{CO}$ & 78 & Ilaje & 0.7 \\
\hline & & VOC & 1.2 & Ilaje & 0.02 \\
\hline \multirow{5}{*}{14} & \multirow{5}{*}{$\begin{array}{l}\text { Qua Petroleum Refinery } \\
\text { Limited } \\
\text { (100,000 bpsd })\end{array}$} & $\mathrm{PM}_{10}$ & 1.0 & Ibeno & 0.4 \\
\hline & & $\mathrm{SO}_{2}$ & 142.5 & Ibeno & $24.0^{\mathrm{a}}$ \\
\hline & & $\mathrm{NO}_{\mathrm{x}}$ & 623 & Ibeno & $8.3^{\mathrm{a}}$ \\
\hline & & $\mathrm{CO}$ & 423 & Ibeno & 3.7 \\
\hline & & VOC & 6.5 & Ibeno & 0.1 \\
\hline
\end{tabular}




\section{Continued}

\begin{tabular}{|c|c|c|c|c|c|}
\hline \multirow{5}{*}{15} & \multirow{5}{*}{$\begin{array}{l}\text { Rehoboth Natural } \\
\text { Resources Limited } \\
\quad(12,000 \text { bpsd }\end{array}$} & $\mathrm{PM}_{10}$ & 0.05 & Yenegoa & 0.02 \\
\hline & & $\mathrm{SO}_{2}$ & 2.4 & Yenegoa & 92.3 \\
\hline & & $\mathrm{NO}_{\mathrm{x}}$ & 12.1 & Yenegoa & 16.1 \\
\hline & & $\mathrm{CO}$ & 9.7 & Yenegoa & 0.1 \\
\hline & & VOC & 0.2 & Yenegoa & 0.003 \\
\hline \multirow{5}{*}{16} & \multirow{5}{*}{$\begin{array}{l}\text { Resources Refinery \& } \\
\text { Petrochemical Limited } \\
\quad(100,000 \text { bpsd })\end{array}$} & $\mathrm{PM}_{10}$ & 1.3 & Ikot Abasi & 0.7 \\
\hline & & $\mathrm{SO}_{2}$ & 191.2 & Ikot Abasi & $8.6^{\mathrm{a}}$ \\
\hline & & $\mathrm{NO}_{\mathrm{x}}$ & 897.3 & Ikot Abasi & $14.8^{\mathrm{a}}$ \\
\hline & & $\mathrm{CO}$ & 399.4 & Ikot Abasi & 5.8 \\
\hline & & VOC & 6.0 & Ikot Abasi & 0.2 \\
\hline \multirow{5}{*}{17} & \multirow{5}{*}{$\begin{array}{l}\text { RIVGAS Petroleum \& } \\
\text { Energy Limited } \\
\text { (30,000 bpsd })\end{array}$} & $\mathrm{PM}_{10}$ & 0.3 & Port Harcourt & 0.1 \\
\hline & & $\mathrm{SO}_{2}$ & 0.4 & Port Harcourt & 1.5 \\
\hline & & $\mathrm{NO}_{\mathrm{x}}$ & 112.3 & Port Harcourt & $1.5^{\mathrm{a}}$ \\
\hline & & $\mathrm{CO}$ & 3.9 & Port Harcourt & 0.03 \\
\hline & & VOC & 1.8 & Port Harcourt & 0.02 \\
\hline \multirow{5}{*}{18} & \multirow{5}{*}{$\begin{array}{l}\text { Sapele Refinery Limited } \\
\quad(100,000 \text { bpsd })\end{array}$} & $\mathrm{PM}_{10}$ & 0.3 & Sapele & 0.1 \\
\hline & & $\mathrm{SO}_{2}$ & 48.9 & Sapele & $18.8^{\mathrm{a}}$ \\
\hline & & $\mathrm{NO}_{\mathrm{x}}$ & 255.2 & Sapele & $3.4^{\mathrm{a}}$ \\
\hline & & $\mathrm{CO}$ & 48.9 & Sapele & 0.4 \\
\hline & & VOC & 1.6 & Sapele & 0.03 \\
\hline \multirow{4}{*}{19} & \multirow{4}{*}{$\begin{array}{l}\text { South West Refinery \& } \\
\text { Petrochemical Company } \\
(100,000 \text { bpsd })\end{array}$} & $\begin{array}{l}\mathrm{PM}_{10} \\
\mathrm{SO}_{2}\end{array}$ & $\begin{array}{c}0.7 \\
59.4\end{array}$ & $\begin{array}{l}\text { Ogun water side } \\
\text { Ogun water side }\end{array}$ & $\begin{array}{c}0.3 \\
22.8^{\mathrm{a}}\end{array}$ \\
\hline & & $\mathrm{NO}_{\mathrm{x}}$ & 270 & Ogun water side & $3.6^{\mathrm{a}}$ \\
\hline & & $\mathrm{CO}$ & 149.8 & Ogun water side & 1.3 \\
\hline & & VOC & 2.4 & Ogun water side & 0.04 \\
\hline \multirow{5}{*}{20} & \multirow{5}{*}{$\begin{array}{l}\text { Starrex Petroleum Refinery } \\
\quad(100,000 \text { bpsd })\end{array}$} & $\mathrm{PM}_{10}$ & 1.0 & Eleme & 0.4 \\
\hline & & $\mathrm{SO}_{2}$ & 0.4 & Eleme & 1.5 \\
\hline & & $\mathrm{NO}_{\mathrm{x}}$ & 112.3 & Eleme & $1.5^{\mathrm{a}}$ \\
\hline & & $\mathrm{CO}$ & 3.9 & Eleme & 0.03 \\
\hline & & VOC & 1.8 & Eleme & 0.03 \\
\hline \multirow{5}{*}{21} & \multirow{5}{*}{$\begin{array}{l}\text { Towei Oil Refinery } \\
\text { (200,000 bpsd) }\end{array}$} & $\mathrm{PM}_{10}$ & 1.5 & Ekeremor & 0.6 \\
\hline & & $\mathrm{SO}_{2}$ & 376 & Ekeremor & $1.0^{\mathrm{a}}$ \\
\hline & & $\mathrm{NO}_{\mathrm{x}}$ & 1875.4 & Ekeremor & $2.5^{\mathrm{a}}$ \\
\hline & & $\mathrm{CO}$ & 168.9 & Ekeremor & 1.5 \\
\hline & & VOC & 1.6 & Ekeremor & 0.03 \\
\hline
\end{tabular}




\begin{tabular}{|c|c|c|c|c|c|}
\hline \multicolumn{6}{|c|}{ Continued } \\
\hline \multirow{5}{*}{22} & \multirow{5}{*}{$\begin{array}{l}\text { Total Support Limited } \\
\quad(12,000 \text { bpsd })\end{array}$} & $\mathrm{PM}_{10}$ & 0.7 & Calabar Municipal & 0.3 \\
\hline & & $\mathrm{SO}_{2}$ & 2.9 & Calabar Municipal & $6.2^{\mathrm{a}}$ \\
\hline & & $\mathrm{NO}_{\mathrm{x}}$ & 16 & Calabar Municipal & 21.3 \\
\hline & & $\mathrm{CO}$ & 22 & Calabar Municipal & 0.2 \\
\hline & & VOC & 0.5 & Calabar Municipal & 0.01 \\
\hline \multirow{5}{*}{23} & \multirow{5}{*}{$\begin{array}{l}\text { Union Atlantic Petroleum } \\
\text { Refinery }(100,000 \text { bpsd })\end{array}$} & $\mathrm{PM}_{10}$ & 1.8 & Badagry & 0.7 \\
\hline & & $\mathrm{SO}_{2}$ & 224 & Badagry & $86.2^{\mathrm{a}}$ \\
\hline & & $\mathrm{NO}_{\mathrm{x}}$ & 116.8 & Badagry & $1.6^{\mathrm{a}}$ \\
\hline & & $\mathrm{CO}$ & 658 & Badagry & 5.8 \\
\hline & & VOC & 10.3 & Badagry & 0.2 \\
\hline
\end{tabular}

${ }^{a}$ folds of $\%$ set limits of the standard (above the set standard limit).

Table 6. Cumulative ground level concentration impact on ambient quality of multiple refineries within each state.

\begin{tabular}{|c|c|c|c|c|}
\hline $\mathrm{S} / \mathrm{N}$ & Refinery & Air Pollutant & $\begin{array}{l}\text { Cumulative Maximum } \\
\text { glc }\left(\mu \mathrm{g} / \mathrm{m}^{3}\right)\end{array}$ & $\begin{array}{l}\text { \% Set Limit of FMENV, } \\
\text { WB, WHO }\end{array}$ \\
\hline \multirow{5}{*}{1} & \multirow{5}{*}{$\begin{array}{c}\text { Rehoboth Natural Resources Limited } \\
\& \\
\text { Towei Oil Refinery } \\
\text { Bayelsa State }\end{array}$} & $\mathrm{PM}_{10}$ & 1.5 & 0.6 \\
\hline & & $\mathrm{SO}_{2}$ & 376 & $14.5^{\mathrm{a}}$ \\
\hline & & $\mathrm{NO}_{\mathrm{x}}$ & 1875.4 & $25.0^{\mathrm{a}}$ \\
\hline & & $\mathrm{CO}$ & 168.9 & 1.5 \\
\hline & & VOC & 1.6 & 0.03 \\
\hline \multirow{5}{*}{2} & \multirow{5}{*}{$\begin{array}{l}\text { Total Support Limited \& Amexum Corporation } \\
\text { Cross River State }\end{array}$} & $\mathrm{PM}_{10}$ & 0.5 & 0.2 \\
\hline & & $\mathrm{SO}_{2}$ & 7.0 & $2.7^{\mathrm{a}}$ \\
\hline & & $\mathrm{NO}_{\mathrm{x}}$ & 314.4 & $4.2^{\mathrm{a}}$ \\
\hline & & $\mathrm{CO}$ & 215 & 1.9 \\
\hline & & VOC & 2.3 & 0.1 \\
\hline \multirow{5}{*}{3} & \multirow{5}{*}{$\begin{array}{c}\text { Antonio Oil, South West Refinery \& Gasoline International } \\
\text { Limited Refinery } \\
\text { Ogun State }\end{array}$} & $\mathrm{PM}_{10}$ & 4.6 & 1.8 \\
\hline & & $\mathrm{SO}_{2}$ & 107.4 & $4.1^{\mathrm{a}}$ \\
\hline & & $\mathrm{NO}_{\mathrm{x}}$ & 426.4 & $5.7^{\mathrm{a}}$ \\
\hline & & $\mathrm{CO}$ & 149.8 & $1.7^{\mathrm{a}}$ \\
\hline & & VOC & 2.6 & 0.04 \\
\hline \multirow{5}{*}{4} & \multirow{5}{*}{$\begin{array}{l}\text { Ode Aye Refinery Limited, Ilaje Refinery \& } \\
\text { Petrochemicals \& Owena Oil \& Gas Limited } \\
\text { Ondo state }\end{array}$} & $\mathrm{PM}_{10}$ & 0.8 & 0.3 \\
\hline & & $\mathrm{SO}_{2}$ & 84.5 & $3.3^{\mathrm{a}}$ \\
\hline & & $\mathrm{NO}_{\mathrm{x}}$ & 444.5 & $5.9^{\mathrm{a}}$ \\
\hline & & $\mathrm{CO}$ & 199.5 & 1.8 \\
\hline & & VOC & 3.0 & 0.05 \\
\hline \multirow{5}{*}{5} & \multirow{5}{*}{$\begin{array}{c}\text { Warri Refining, Niger Delta Refinery \& Sapele Refinery } \\
\text { Limited } \\
\text { Delta State }\end{array}$} & $\mathrm{PM}_{10}$ & 1.7 & 0.7 \\
\hline & & $\mathrm{SO}_{2}$ & 443.3 & $17.1^{\mathrm{a}}$ \\
\hline & & $\mathrm{NO}_{\mathrm{x}}$ & 2145.8 & $28.6^{\mathrm{a}}$ \\
\hline & & $\mathrm{CO}$ & 480.3 & 4.2 \\
\hline & & VOC & 7.0 & 0.1 \\
\hline
\end{tabular}




\begin{tabular}{|c|c|c|c|c|}
\hline \multicolumn{5}{|c|}{ Continued } \\
\hline \multirow{5}{*}{6} & \multirow{4}{*}{$\begin{array}{c}\text { Amakpe International Refinery, Chase Wood Consortium } \\
\text { Nigeria Limited, Resources Refinery \& Qua Petroleum } \\
\text { Refinery Limited } \\
\text { Akwa Ibom State }\end{array}$} & $\mathrm{PM}_{10}$ & 8.0 & 3.2 \\
\hline & & $\mathrm{SO}_{2}$ & 501 & $19.3^{\mathrm{a}}$ \\
\hline & & $\mathrm{NO}_{\mathrm{x}}$ & 2214 & $29.5^{\mathrm{a}}$ \\
\hline & & $\mathrm{CO}$ & 480.3 & 3.7 \\
\hline & & VOC & 7.0 & 0.4 \\
\hline \multirow{5}{*}{7} & & $\mathrm{PM}_{10}$ & 0.2 & 0.4 \\
\hline & $\begin{array}{l}\text { Clean Water Refinery, NSP Refineries and Oil Services } \\
\text { Limited, RIVGAS Petroleum \& Energy Limited, Starrex }\end{array}$ & $\mathrm{SO}_{2}$ & 204 & $7.8^{\mathrm{a}}$ \\
\hline & $\begin{array}{l}\text { Petroleum Refinery, Port Harcourt Refining Company I \& } \\
\text { Port Harcourt Refining Company II }\end{array}$ & $\mathrm{NO}_{\mathrm{x}}$ & 957 & $12.8^{\mathrm{a}}$ \\
\hline & River state & $\mathrm{CO}$ & 1.6 & 3.5 \\
\hline & & VOC & 0.1 & 0.1 \\
\hline
\end{tabular}

${ }^{\mathrm{a}}$ folds of $\%$ set limits of the standard i.e. above the set standard limit.

four existing refineries and twenty-three proposed refineries in Nigeria for the purpose of predicting the air quality impact. Emissions from minimum of seven and maximum of eleven elevated point sources of criteria pollutants with "worst case" scenario were considered. The 24-hr averaging period of ground level concentration was measured and compared with the Nigeria's ambient quality air set limits as set by National Ambient Air Quality Standard (NAAQS), World Bank and World Health Organization (WHO) within $50 \mathrm{~km}$ receptor locations.

From the modeling outputs, $\mathrm{NO}_{\mathrm{x}}$ and $\mathrm{SO}_{2}$ are the major pollutants that exceed the set limit, hereby causing harmful effects to the environment while PM, NOx and VOCs are within the set standard and have no effect on the environment. The highest ground level concentration predicted to be $450-1875 \mu \mathrm{g} / \mathrm{m}^{3}$ for $24-\mathrm{h}$ averaging period was obtained at Tonwei Oil Refinery, Ekeremor Local Government, Bayelsa State, proposed to operate at installed capacity 200,000 barrels/day capacity while the lowest ground level concentration predicted to be $0.0099-0.1 \mu \mathrm{g} / \mathrm{m}^{3}$ for 24-h averaging period was obtained at Amakpe International Refinery, Eket Local Government, Akwa Ibom State, proposed to operate at installed capacity 12,000 barrels/day. According to Ambient Air Quality Standards (AAQS), percentage set limits of criteria air pollutants ranging from $0.02 \%-94.5 \%$ are within the set standard limits and no health risk is associated with areas around the plant locations while percentage set limits of criteria pollutants ranging from 1.1 - 55.6 folds of the standards exceed the maximum permitted limits and health risk is associated with areas around the plant locations as stipulated by the National Ambient Air Quality Standard (NAAQS), World Bank and World Health Organization (WHO) emission set standards.

The study reveals that the contribution of emissions from criteria pollutant to its ambient air is significant. Possible impacts of anticipated emissions of criteria pollutants on humans [17], vegetations [18] soil and water [19] [20] are strong enough for necessary control measures to be implemented in both existing and proposed Nigerian petroleum refineries. Hence, the air quality standards guiding petroleum refinery emissions must be strictly considered, in order to ensure that the ground level concentrations do not exceed the required standard limits and prevent the adverse effect of air pollution in the Nigeria airshed.

\section{References}

[1] [USEPA] United State Environmental Protection Agency (1999) Proper Monitoring Essential to Reducing Fugitive Emissions under Leak Detection and Repair Programs. Enforcement Alert, 2, 1-4.

[2] [WB] World Bank (1998) Petroleum Refining. Pollution Prevention and Abatement Handbook. World Bank Group, 3, 377-380.

[3] [WHO] World Health Organization (2005) WHO Air Quality Guidelines for Particulate Matter, Ozone, Nitrogen Dioxide and Sulphur Dioxide Global Update. Summary of Risk Assessment, 23, 1-20.

[4] Zannetti, P., Ed. (1994) Environmental Systems. Proceedings of ENVIROSOFT 94 Conference, Vol. II, San Francisco, November 1994, Computational Mechanics Publications, Southampton. http://www.witpress.com/

[5] Schnelle, K.B. and Dey, P.R. (1999) Atmospheric Dispersion Modeling Compliance Guide McGraw-Hill Professional. ISBN 0-07-058059-6. 
[6] Elkamel, A., Abdul-Wahab, S., Bouhamra, W. and Alper, E. (2001) Measurement and Prediction of Ozone Levels around a Heavily Industrialized Area: A Neural Network Approach. Environmental Research, 5, 47-49. http://dx.doi.org/10.1016/S1093-0191(00)00042-3

[7] Sonibare, J.A. and Ede, P.N. (2009) Potential Impacts of Integrated Oil and Gas Plant on Ambient Air Quality. Energy \& Environment, 20, 331-344. http://dx.doi.org/10.1260/095830509788066394

[8] [DPR] Department of Petroleum Resource (2001) Nigeria Oil Industry Statistical Bulletin. Department of Petroleum Resources, Ministry of Petroleum Resources, Lagos.

[9] [DPR] Department of Petroleum Resource (2004) Nigeria Oil Industry Statistical Bulletin. Department of Petroleum Resources, Ministry of Petroleum Resources, Lagos.

[10] [DPR] Department of Petroleum Resource (2010) Private Refineries and Petrochemical Plants Status. Nigeria Oil Industry Statistical Bulletin. Department of Petroleum Resources, Ministry of Petroleum Resources, Lagos.

[11] [FEPA] Federal Environmental Protection Agency (1991) National Interim Guideline and Standards for Industrial Effluents. Gaseous Emissions and Harzadous Wastes, 5, 33-63.

[12] [WB] World Bank (1998) Petroleum Refining. In: World Bank, Ed., Pollution Prevention and Abatement Handbook, 1998: Toward Cleaner Production, World Bank Group, Washington DC, 377-380.

[13] [WHO] World Health Organization (2005) WHO Air Quality Guidelines for Particulate Matter, Ozone, Nitrogen Dioxide and Sulphur Dioxide. Global Update 2005, Summary of Risk Assessment, 23, 1-14.

[14] Abdul-Wahab, S.A. and Al-Alawi, S. (2002) Assessment and Prediction of Tropospheric Ozone Concentration Levels Using Artificial Neural Networks. Environmental Modelling Software, 17, 219-228. http://dx.doi.org/10.1016/S1364-8152(01)00077-9

[15] Chang, T. and Stell, J. (2000) Worldwide Refining Survey. Oil Gas, 66-120.

[16] [USEPA] United States Environmental Protection Agency (1989) Airs Facility Subsystem Source Codes and Emission Factor Listing for Criteria Air Pollutants. Research Triangle Park, N.C. U.S. Environmental Protection Agency.

[17] Gou, H., Lee, S.C., Chan, L.Y. and Li, W.M. (2004) Risk Assessment of Exposure to Volatile Organic Compounds in Different Indoor Environments. Environmental Research, 94, 57-66. http://dx.doi.org/10.1016/S0013-9351(03)00035-5

[18] Rao, P.S., Gavane, A.G., Ankam, P., Ansari, M.F., Pandit, V.I. and Nema, P. (2004) Performance Evaluation of a Green Belt in a Petroleum Refinery: A Case Study. Ecological Engineering, 23, 77-84. http://dx.doi.org/10.1016/j.ecoleng.2004.06.013

[19] Johnson, R.L., Thomas, T.B. and Zogorski, J.J. (2003) Effects of Daily Precipitation and Evapotranspiration Patterns on Flow and VOC Transport to Groundwater along a Watershed Flow Path. Environmental Science Technology, 37, 4944-4954. http://dx.doi.org/10.1021/es026252c

[20] Kim, H., Annabel, M.D. and Rao, P.S. (2001) Gaseous Transport of Volatile Organic Chemicals in Unsaturated Porous Media: Effect of Water Partitioning and Air-Water Interfacial Adsorption. Environmental Science Technology, 35, 4457-4462. http://dx.doi.org/10.1021/es0019651 